\title{
Die Verführung durch Daten
}

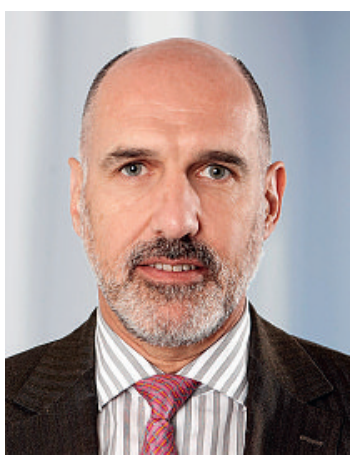

Das Parlament hat die «Massnahmen zur Eindämmung der Kostenentwicklung» im Oktober abgelehnt - und damit unter anderen den Artikel, der die systematische Übermittlung von Diagnosedaten an die Versicherer verlangte. Die FMH hatte sich, wie auch $\mathrm{H}+$, mehrmals gegen diese Vorschrift und damit die Abschaffung des Arztgeheimnisses in Briefen an die Parlamentarier und in persönlichen Kontakten gewehrt.

Trotz der finalen Ablehnung stellt man fest, dass der Datenhunger durch die elektronische Datenübermittlung und -bearbeitung anhält und gar zunimmt - getreu dem Irrtum, mehr Daten würden mehr Wissen, damit letztendlich mehr Kontrollmöglichkeiten bedeuten.

Die FMH fördert seit langem die Umsetzung von eHealth als Mittel zur Unterstützung und Optimierung der Patientenbehandlung; eHealth ist ein wichtiges Instrument, Behandlungsprozesse besser sowie sicherer zu gestalten, und trägt auch zur Umsetzung neuer integrierter Behandlungskonzepte bei.

Nur, wir erfahren es jeden Tag wieder: Für eine erfolgreiche Behandlung müssen sich Patienten uns vorbehaltlos anvertrauen können und wissen, dass das von ihnen Gesagte

\section{SwissDRG: Kontrolle muss über externe professionelle Kodierrevision erfolgen}

nicht weitergegeben wird. Dieses Vertrauen kann weder durch eine Verpflichtung zur elektronischen Dokumentation, noch durch geringere Versicherungsprämien erzwungen werden. Man kann es nur aufbauen und halten, indem der Arzt die ihm anvertrauten Informationen adäquat schützt.

Der Datenhunger der Versicherer ist aber damit häufig nicht kompatibel, und wir werden uns in der Zukunft immer mehr dafür einsetzen müssen, dass das Arztgeheimnis nicht zu einer Worthülse wird.

Im stationären Bereich sind für die Überprüfung von einzelnen Spitälern keine detaillierten Daten auf Einzelrechnungen erforderlich. Die Medizinische Statistik des Bundesamtes für Statistik ist der Beweis dafür, dass Profile von Spitälern auch mit anonymisierten Daten erstellt werden können.
Auch die FMH befürwortet eine angemessene Kontrolle der Codierung bei DRG-Rechnungen. Diese kann jedoch nur gestützt auf die Krankengeschichte erfolgen - dafür ist eine externe professionelle Kodierrevision das geeignete Instrument: extern, weil sie durch einen unabhängigen Revisor durchgeführt wird; professionell, weil die Revisoren dafür

\section{Auch Angaben auf Spitex- und Heimrechnungen verletzen die Intim- sphäre und das Selbstbestimmungs- recht des Patienten}

qualifiziert sind. Diese Kontrolle ist durch die von den Tarifpartnern gemeinsam beauftragten externen Revisoren durchzuführen. Völlig undenkbar ist, Kontrolleuren einer einzelnen Kasse Zugang zu den Krankengeschichten zu geben!

Auch in der ambulanten Medizin muss man zwischen Mittel und Zweck unterscheiden! Die Versicherer wollen neben der ihnen zustehenden Aufgabe der Kostenkontrolle zunehmend auch die eigentliche Behandlungsführung kontrollieren. Ihre Bemühungen, die Diagnosen aller Patienten auf jeder Rechnung zu erzwingen, geht in diese Richtung. Die Angabe von detaillierten ICD-Diagnosen, wie es im eingangs erwähnten Massnahmenpaket vorgesehen war, hätte erheblichen administrativen Zusatzaufwand und Zusatzkosten verursacht. Zudem können im ambulanten Bereich Diagnosen in einem solchen Detaillierungsgrad häufig überhaupt nicht gestellt werden. Vor allem aber hätte man das Arztgeheimnis faktisch aufgehoben.

Eine Rechnung gelangt an Verwaltungsangestellte einer Versicherung und muss dort über Jahre aufbewahrt werden. Sie ist somit unzähligen unbekannten Personen zugänglich. Je nach Wohn- und sozialer Situation gelangt die Rechnung aber zudem auch in die Hände von Familienangehörigen und von Sozialhilfe-, Vormundschafts- oder Steuerbehörden. Auch diese dürfen nicht alle erfahren, welche Infektionen eine Person hatte oder aufgrund welcher Diagnose sie in psychiatrischer Behandlung ist. Darüber hinaus können Spitex- und Heimrechnungen die Intimsphäre und das informationelle Selbstbestimmungsrecht des Patienten verletzen wie etwa mit Angaben über Demenz oder Inkontinenz.

Es wird also für die FMH in der Zukunft ein zentrales Anliegen bleiben, den Datenhunger von Versicherern in Grenzen zu halten. Die Grundsätze der Medizin, in diesem Fall das Arztgeheimnis, sind nicht verhandelbar!

Dr. med. Jacques de Haller, Präsident der FMH 